\title{
Painful Cutaneous and Subcutaneous Tumors Accompanied with Vascularized Appearance using High-Resolution Ultrasound in Dermatology: The Acronym "ENGLAND" or "LEND AN EGG"
}

\author{
Kazumi Fujioka* \\ Division of Laboratory Medicine, Department of Pathology and Microbiology, Nihon University School of Medicine, Tokyo, Japan
}

Received: 盋: December 12, 2018; Published: 䟧: January 03, 2019

*Corresponding author: Kazumi Fujioka, Division of Laboratory Medicine, Department of Pathology and Microbiology, Nihon University School of Medicine, Tokyo, Japan

\begin{abstract}
The acronym "ENGLAND" or "LEND AN EGG" is often used to recall these painful tumors in dermatology. "LEND AN EGG" tumors show: leiomyoma, eccrine spiradenoma, neuroma, dermatofibroma, angiolipoma, neurilemmoma, endometrioma, glomus tumor, and granular cell tumor. Dermatologic ultrasound imaging has been rapidly growing in recently years because of the development of high-resolution multifrequency transducers and multichannel color Doppler machines. All painful cutaneous and subcutaneous tumors in dermatology used the acronym "ENGLAND" especially, angioleiomyoma, eccrine spiradenoma, schwannoma and glomus tumor show vascularized features on high-resolution US. Blood flow signals is more detectable in schwannoma than in neuroma. Angioleiomyoma mimic schwannoma very closely. The features of nerve-tumor association and adjacent vessel could be useful for differentiation of between schwannoma and angioleiomyoma.
\end{abstract}

Keywords: ENGLAND; LEND AN EGG; Dermatologic Ultrasound; High-Resolution Ultrasonography; Color Doppler Ultrasonography; Painful And Vascularized Tumor

Abbreviations: DERMUS: Dermatologic Ultrasound; RTE: Real-time Tissue Elastography ; ALM: Angioleiomyoma

\section{Introduction}

The acronym "ENGLAND" or "LEND AN EGG" is often used to recall these painful tumors in dermatology. "ENGLAND" tumors show: eccrine spiradenoma, neuroma, glomus tumor, leiomyoma, angiolipoma, neurilemmoma, and dermatofibroma. "LEND AN EGG" tumors show: leiomyoma, eccrine spiradenoma, neuroma, dermatofibroma, angiolipoma, neurilemmoma, endometrioma, glomus tumor, and granular cell tumor [1]. The acronym "LEND AN EGG" show "ENGLAND” entities associated with endometrioma and granular cell tumor. Dermatologic ultrasound imaging has been rapidly growing in recently years because of the development of high-resolution multifrequency transducers and multichannel color Doppler machines [2]. The international working group, called DERMUS (Dermatologic Ultrasound) was formed and provided the guidelines for performing dermatologic ultrasound examinations [2] and proposed for an assessment training program
$[2,3]$. The minimum frequency recommended for performing dermatologic examinations was $15 \mathrm{MHz}[2,3]$. We usually perform US examinations for dermatologic diseases with a high-resolution, broad-band (5MHz-18MHz) linear transducer (Nobulus Hitachi, Ltd.Tokyo, Japan) and have described some reports [4-9].

Meanwhile, vascularized superficial benign soft-tissue tumors on sonography with a histopathological correlation are regarded as eccrine spiradenoma, dermatofibroma, schwannoma, vascular leiomyoma, glomus tumor, pilomatricoma, chondroid syringoma, hemangioma [10]. Many of painful tumors [7,11-17] show vascularized features on color Doppler US. Vascular analysis is considered as vascular pattern, vascular density and vascular distribution $[17,18]$. In this article, I review vascularized image features using high-resolution color Doppler US in relation to the acronym "ENGLAND" or "LEND AN EGG" showing painful tumors in dermatology. 


\section{The Acronym “ENGLAND” or "LEND AN EGG” Entities}

Eccrine Spiradenoma: Eccrine spiradenoma are rare benign tumors of the sweat gland, showing hererogeneously hypoechoic with lobular margin on gray-scale US and with increased peripheral portion, with or without blood flow in the central portion on color Doppler US [11]. Real-time Tissue Elastography (RTE) is widely used for diagnosis of breast and thyroid lesions on tissue elasticity. Recently, it has been reported that the usefulness of elastography is described in dermatologic field [7,9]. Sonoelastography evaluation showed a predominantly blue color, suggesting hard tissue component [19].

Neuromas: Benign neurogenic tumors affecting the musculoskeletal system include schwannoma, neurofibromas, traumatic neuromas, Morton neuromas, neural fibrolipomas, and nerve sheath ganglion cysts [12]. Neuromas include both the painful amputation and Morton's varieties, which differ clinically and histologically [1]. Regarding the neurofibromas, nerve-tumor position shows central position on gray-scale US. Neurofibroma grow interstitially in the center of the nerve bundle within the endoneurium and are not encapsulated, resulting in the infiltrative condition of the nerve-tumor transition [12]. It has been reported that most of the neurofibromas were hyopovascular condition [12].

Glomus Tumors: Glomus tumors may be spontaneously painful. They may be purple or blue-red and occur commonly in the nail bed [1]. Glomus tumors appears as well-defined hypoechoic nodules with a profuse hypervascular component accompanied by active bone remodeling of the distal phalanx on gray-scale and color Doppler US [16]. On the other hand, diffusely scattered irregular vessels are depicted on color Doppler US and spectral analysis demonstrated high speed and high resistance profile in no subungual glomus tumors [17].

Leiomyoma: Cutaneous leiomyoma are uncommon benign smooth muscle neoplasms of skin. Three variants of this tumor can be found in the skin as piloleiomyoma, angioleiomyoma (ALM) and genital leiomyoma. ALM is a rare tumor that arises from the tunica media of small arteries and veins. ALM presents clinically as a solitary, firm, well-circumscribed tumor measuring $<20 \mathrm{~mm}$ in size. Histologically, ALM shows a proliferation of smooth muscle cells and vascular channels. Based on the size of the vascular channel and the degree of muscular thickness, ALM have been classified into three histological subtypes: a solid or capillary type, a cavernous type and a venous type. The solid subtype of ALM usually presents as a painful tumor in the limbs and occurs more frequently in females [20]. Recently, we have reported an ALM of the thigh in female with intermittent pain using high-resolution ultrasound including gray-scale US, color Doppler US and Real-Time Tissue Elastography (RTE). Vascular distribution was regarded as central and peripheral pattern on color Doppler US and peripheral flow signals was suggested to be consistent with adjacent vessel as previously described [7].

Angiolipoma: Angiolipomas can be painful and are one of the varieties of lipomas. Fibrin thrombi may be found within capillar- ies, and the degree of vascular proliferation may or may not be associated with pain. Bang et al reported that most subcutaneous angiolipomas are oval-shaped, well-defined margins, and hyperechoic appearance on gray-scale US. They also described that color Doppler US findings may help in differentiating angiolipoma from ordinary subcutaneous lipoma. Shin, et al also reported that sonography might help differentiate angiolipoma from superficial lipomas.

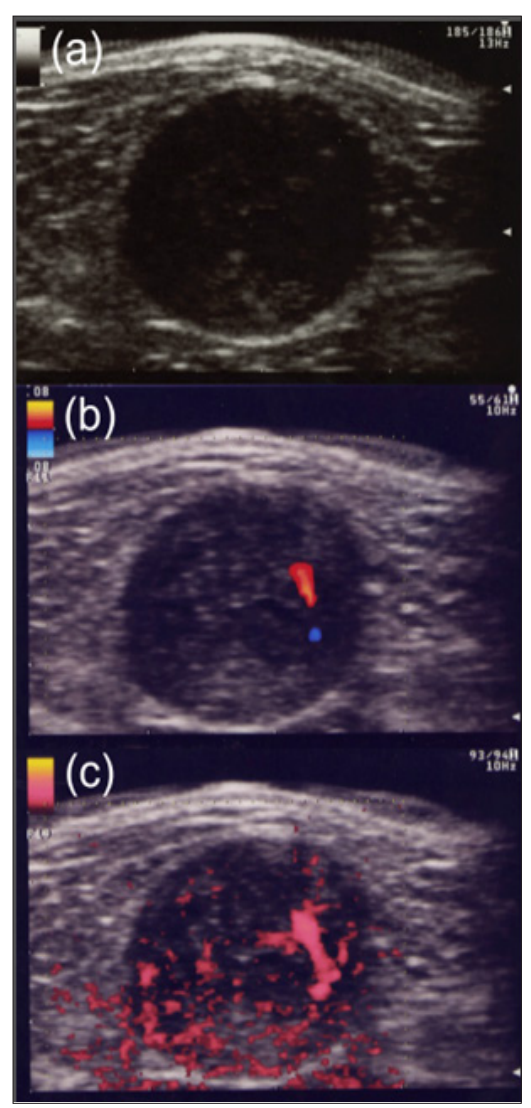

Figure 1: Schwannoma pathologically confirmed in the thigh in a 47-year-old male.

a) On gray-scale US show a well-defined hypoechoic nodule with cystic change and eccentric nerve-tumor position.

b) A few central blood flow signals are depicted on color Doppler US.

c) Power Doppler US show many central vascularity in a mass.

Neurilemmomas or Schwannoma: Neurilemmoma, or schwannomas, occur along the course of a peripheral nerve. They may be tender and firm and have a flash- to pale-pink color. Histologically, S-shaped nuclei, Verocay bodies are characteristic. Both Antoni A and Antoni B tissue are present. The nerve-tumor association and vascularity of the tumors could be useful for differentiation of between schwannoma and neuroma. Schwannoma shows eccentric nerve-tumor position on gray-scale US and vascularity within the tumor on color Doppler US [12]. Tsai et al. reported that color Doppler US shows hypervascular flow signals in a patient with schwannoma [21]. US findings of pathologically 
confirmed as a schwannoma in the thigh in a 47-year-old male were present as follows: A well-defined hypoechoic nodule with cystic change and eccentric nerve-tumor position were depicted on grayscale US. Blood flow signals were more detectable on power Doppler US than on color Doppler US (Figure 1a-1c). Photomicrograph shows well-defined ovoid mass with degenerative cystic foci. Less cellular Antony type B area consisting of neuronal spindle cells forming Verocay bodies was detected (Figure 2a- 2c). Tumors were comprehensively diagnosed as schwannomas in the upper extremity in a 46-year-old female were noted as follows: The tumor presents as well-defined hypoechoic nodule without an obviously nerve-tumor position on gray-scale US. Many central blood flow signals were depicted both on color Doppler US and power Doppler US (Figure 3a-3c). Figure 4 shows magnetic resonance angiography image as vascular tumors, suggesting schwannomas. ALM mimic schwannoma very closely. The features of nerve-tumor association and adjacent vessel could be useful for differentiation of between schwannoma and ALM as previously described [7].

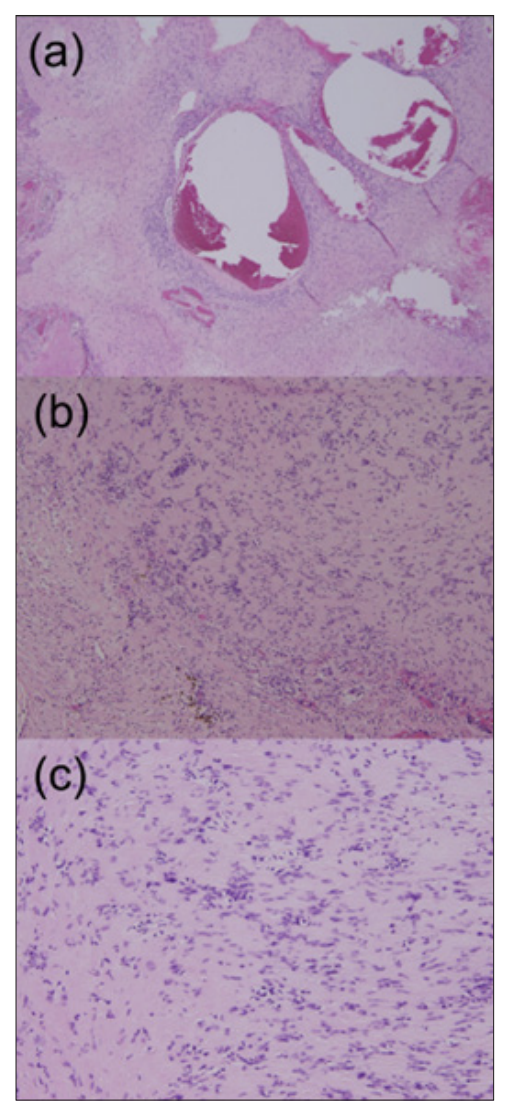

Figure 2: Schwannoma pathologically confirmed in the thigh in a 47-year-old male.

a) Photomicrograph shows well-defined ovoid mass with degenerative cystic foci (hematoxylin and eosin, original magnification $\times 40$ ).

b) Photomicrograph shows less cellular Antony type B (hematoxylin and eosin, original magnification x100).

c) Less cellular Antony type B area consisting of neuronal spindle cells forming Verocay bodies is detected (hematoxylin and eosin, original magnification x200).

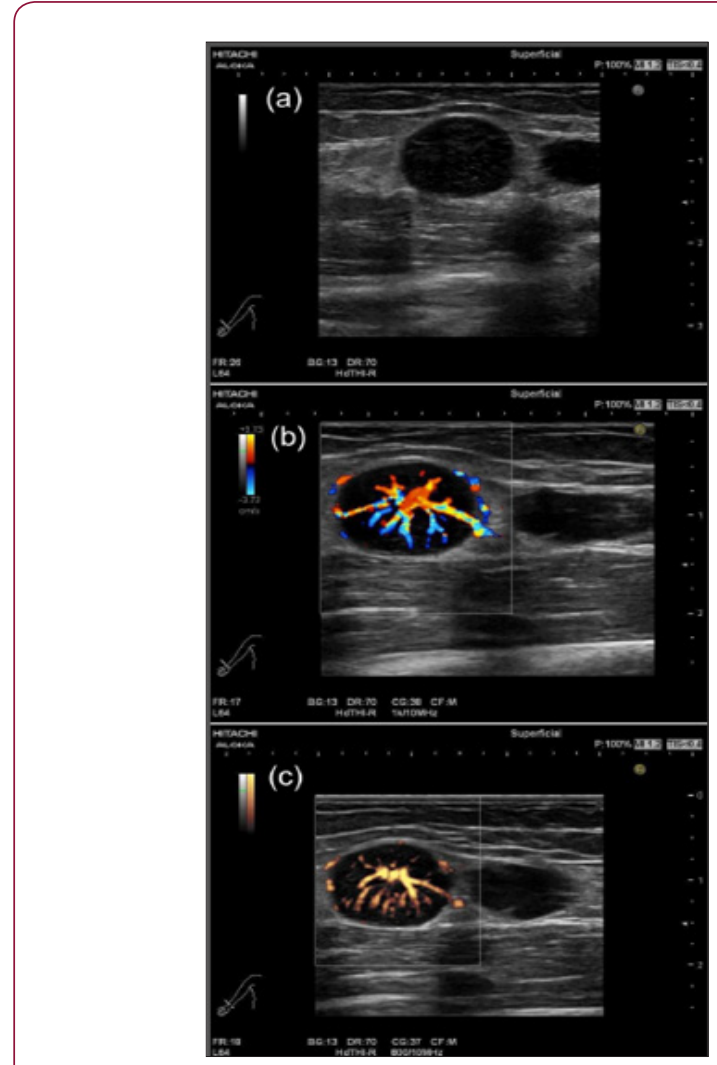

Figure 3: Tumors comprehensively diagnosed as a schwannoma in the upper extremity in a 46-year-old female.

a) The tumor presents as well-defined hypoechoic nodule without an obviously nerve-tumor position on gray-scale US.

b) Many central blood flow signals are depicted on color Doppler US.

c) Power Doppler show central hypervascular flow signals.

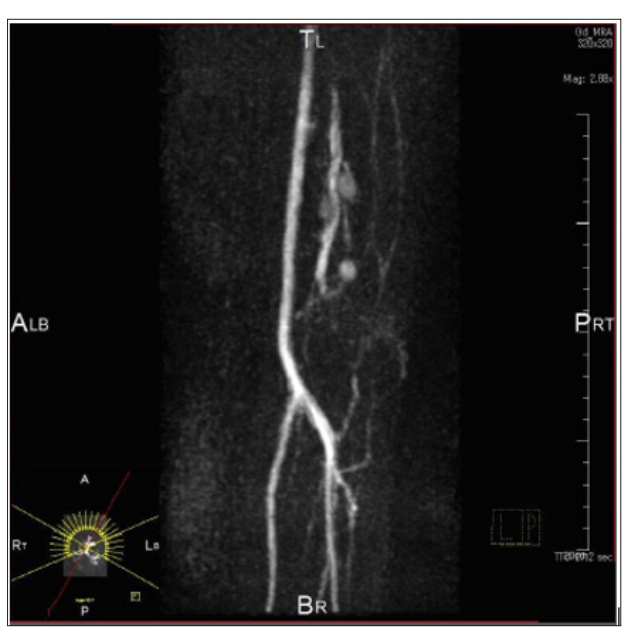

Figure 4: Tumors comprehensively diagnosed as a schwannoma in the upper extremity in a 46-year-old female. Magnetic resonance angiography image shows vascular tumors. 
Dermatofibroma: Although most dermatofibromas are asymptomatic, some are tender. Characteristic epidermal hyperplasia may overlie the tumor, and lipid and hemosiderin may be present in the dermis. Jin, et al. reported that a patient with dermatofibroma showed a well-defined margin and hypoechogenic in the dermis-to-subcutaneous fat layer with increased vascular flow. The acronym "LEND AN EGG" show "ENGLAND" entities associated with endometrioma and granular cell tumor.

Endometriosis or Endometrioma: Endometriosis of the skin is rare, but it may be associated with pain or bleeding that it worse at the time of menstruation. It may develop spontaneously or occur after implantation of endometrial cells in a surgical scar [1].

Granular Cell Tumors: Granular cell tumors may be painful and occur most commonly in the tongue but also in the sin and internal organs [1]. US findings are variable, including a heterogeneous echo texture, an indistinct margin, increased blood flow [15]. All painful tumors in dermatology used the acronym "ENGLAND" especially, angioleiomyoma, eccrine spiradenoma, schwannoma and glomus tumor show vascularized appearances on high-resolution color Doppler US. It has been also reported that granular cell tumors show increased vascularized features. Blood flow signals is more detectable in schwannoma than in neuroma. Schwannoma represent mainly central blood flow signal. Meanwhile, adjacent vascular appearance is characteristic finding of ALM. The classification of the vascular distribution is important in differentiating between schwannoma and angioleiomyoma.

\section{Conclusion}

A. All painful cutaneous and subcutaneous tumors in dermatology used the acronym "ENGLAND" especially, angioleiomyoma, eccrine siradenoma, schwannoma and glomus tumor show vascularized appearances on high-resolution color Doppler US.

B. The classification of the vascular distribution is contributed to the differentiating between schwannoma and angioleiomyoma.

\section{References}

1. Naversen DN, Trask DM, Watson FH, Burket JM (1993) Painful tumors of the skin: “LEND AN EGG". J Am Acad Dermatol 28(2): 298-300.

2. Wortsman X, Alfageme F, Roustan G, Arias Santiago S, Martorell A, et al. (2016) Guidelines for performing dermatologic ultrasound examinations by the DERMUS group. J Ultrasound Med 35(3): 577-580.

3. Wortsman X, Alfageme F, Roustan G, Arias Santiago S, Martorell A, et al. (2016) Proposal for an assessment training program in dermatologic ultrasound by the DERMUS group. J Ultrasound Med 35(11): 2305-2309.

4. Fujioka K, Fujioka A, Eto H, Suzuki K, Sanuki E, et al. (2006) Nodular fascitis in the thigh followed up using ultrasonography. J Med Ultrason 33(1): 49-53.
5. Fujioka K, Fujioka A, Oishi M, Eto H, Tajima S, et al. (2017) Ultrasonography findings of intradermal nodular fasciitis; a rare case report and review of the literature. Clin Exp Dermatol 42(3): 335-336.

6. Fujioka K, Fujioka A, Tajima S, Oishi M, Hayashi K, et al. (2018) Characteristic power Doppler sonographic imaging of nodular fasciitis from a dermatological perspective: another case and review of three cases. J Clin Case Rep 8(9): 1165.

7. Fujioka K, Fujioka A, Oishi M, Hayashi K, Nakayama T (2018) Highresolution ultrasound imaging for angioleiomyoma: a painful and vascularized superficial tumor. Biomed J Sci \& Tec Res 9(5).

8. Fujioka K (2018) Presentations of clinical, ultrasonographic and pathological features of nodular fasciitis from an established cytogenetic viewpoint: review of the cases. J Carcinog Mutagen 9(4).

9. Fujioka K (2018) Usefulness of comprehensive high-resolution ultrasound imaging in dermatologic field: epidermal cyst. Biomed J Sci \& Tec Res 12(1).

10. Jin W, Kim GY, Park SY, Chun YS, Nam DH, et al. (2010) The spectrum of vascularized superficial soft-tissue tumors on sonography with a histopathologic correlation: part 1, benign tumors. AJR 195(2): 439-445.

11. Hwang CM, Kang BS, Hong HJ, LeeJY, Suh JH, et al. (2018) Ultrasonographic features of eccrine spiradenoma. J Ultrasound Med 37(5): 1267-1272.

12. Ryu JA, Lee SH, Cha EY, Kim TV, Kim SM, et al. (2015) Sonographic differentiation between schwannomas and neurofibromas in the susculoskeletal system. J Ultrasound Med 34(12): 2253-2260.

13. Bang M, Kang BS, Hwang JC, Weon YC, Choi SH, et al. (2012) Ultrasonographic analysis of subcutaneous angiolipoma. Skeletal Radiol 41(9): 1055-1059.

14. Shin YS, Kim YJ, Park IS, Chu YC, Kim JH, et al. (2016) Sonographic differentiation between angiolipomas and superficial lipomas. J Ultrasound Med 35(11): 2421-2429.

15. Kim EY, Kang DK, Kim TH, Jung YS, Kim KS, et al. (2011) Granular cell tumor of the male breast: two case descriptions and brief review of the literature. J Ultrasound Med 30(9): 1295-1301.

16. Wortsman X, Wortsman J, Soto R, Saavedra T, Honeyman J, et al. (2010) Benign tumors and pseudotumors of the nail: a novel application of sonography. J Ultrasound Med 29(5): 803-816.

17. Catalano O, Roldan FA, Solivetti FM, Scotto di Santolo M, Bouer M, et al. (2017) Color Doppler sonography of extradigital glomus tumors. J Ultrasound Med 36(1): 231-238.

18. Park HJ, Kim SS, Lee SY, Choi YJ, Chung EC, et al. (2012) Sonographic appearances of soft tissue angioleiomyomas: differences from other circumscribed soft tissue hyper vascular tumors. J Ultrasound Med 31(10): 1589-1595.

19. Balaban M, Idilman IS, Unal O, Dumlu EG, Yazgan A, et al. (2015) Sonographic and sonoelastographic findings of a rarely seen soft tissue tumor: eccrine spiradenoma. J Med Ultrason 42(4): 587-590.

20. Tejasvl BR, Pai K, Rao R (2014) Painful nodule on the leg. Clin Exp Dermatol 39: 542-543.

21. Tsai WC, Chiou HJ, Chou YH, Wang HK, Chiou SY, et al. (2008) Differentiation between schwannomas and neurofibromas in the extremities and superficial body: the role of high-resolution and color doppler ultrasonography. J Ultrasound Med 27(2): 161-166. 


\section{ISSN: 2574-1241}

DOI: $10.26717 / B J S T R .2019 .12 .002297$

Kazumi Fujioka. Biomed J Sci \& Tech Res

(c) (P) This work is licensed under Creative

BY Commons Attribution 4.0 License

Submission Link: https://biomedres.us/submit-manuscript.php

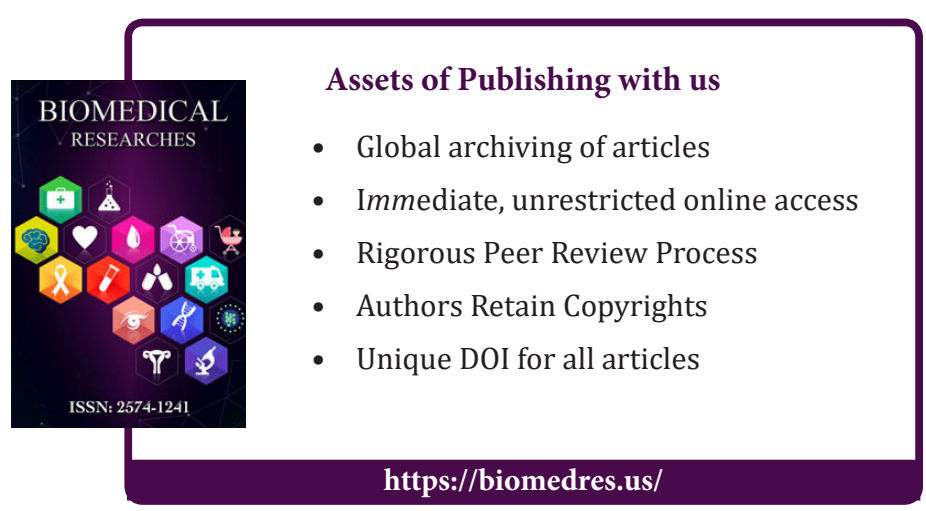

